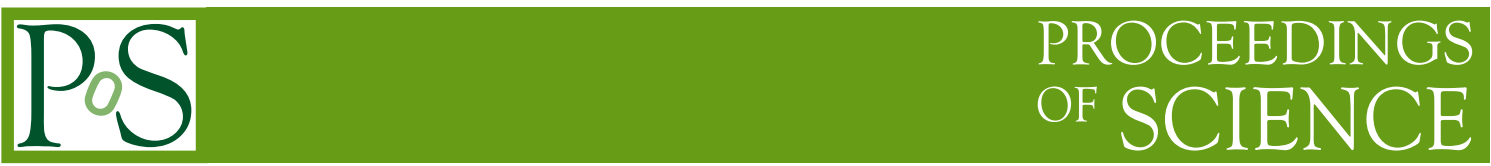

\title{
MeerKAT and the Smallest Galaxies
}

\author{
Elizabeth A. K. Adams* and Tom A. Oosterloo \\ ASTRON, the Netherlands Institute for Radio Astronomy \\ E-mail: adamsdastron.nl, oosterloodastron.nl
}

On small scales, there exists a tension between observations of dwarf galaxies and predictions for low-mass dark matter halos from simulations (often referred to as the small scale crisis). This tension includes a mismatch in number count (e.g., the canonical "missing satellites problem") but also discrepancies in the internal structure (e.g., the "too big too fail" problem). More recently, observations have revealed that low-mass satellite galaxies appear to form structures around their central galaxy ("planes of satellites") while these structures are not predicted in cosmological simulations. Detailed observations of low mass galaxies are critical for constraining the baryonic feedback processes that are used to alleviate these discrepancies. A particularly fruitful course is to study dwarf galaxies that have a substantial reservoir of neutral hydrogen (H I). These are the systems that are the most likely to be isolated, helping to disentangle intrinsic properties from evolutionary effects, and $\mathrm{H}$ I kinematics can offer an immediate constraint on the hosting dark matter halo. Given MeerKAT's exquisite sensitivity, it can potentially contribute to these studies of low-mass H I-rich dwarf galaxies that will help resolve the small scale crisis. The current large H I surveys are not designed for these studies, but will still manage to detect a sample of galaxies with $\mathrm{H}$ I masses below $10^{7} M_{\odot}$ comparable to the number of systems currently in the literature, and will resolve $\sim 15$ systems with masses below $10^{8.5} M_{\odot}$, a critical regime for addressing which dark matter halos host low-mass galaxies. We propose a thousand hour survey of the Centaurus region, encompassing the M 83 and Cen A galaxy groups, which can robustly address key questions in understanding low-mass galaxies. The central question to be addressed is: "How many galaxies equivalent to Leo T are there in the Centaurus region?" Leo T is the lowest mass, gas-rich galaxy currently known; our proposed survey is designed to be able to detect an object of similar H I mass and linewidth throughout the volume of the Centuaurus region, which will provide a full census of how many objects like this there are in a typical galaxy group. In addition, planes of satellites have recently been identified around Cen A and our survey will be able to address how far out these planes extend: are they structures concentrated only around the central galaxy or are they connected to large scale structure?

MeerKAT Science: On the Pathway to the SKA

25-27 May, 2016

Stellenbosch, South Africa

* Speaker. 


\section{The importance of the smallest galaxies}

Studies of dwarf galaxies, especially those with a significant neutral hydrogen (H I) component, offer unique opportunities to test cosmological and galaxy formation models. Living in low-mass dark matter (DM) halos, these systems are the most susceptible to feedback processes and disruption of their baryonic component. Yet they survive, and field dwarfs even retain their gas reservoir. Leo $\mathrm{T}$ is a prime example: this galaxy has an $\mathrm{HI}$ mass of $4.2 \times 10^{5} M_{\odot}$ [1] and a stellar mass of only $10^{5} M_{\odot}$ [2]. This galaxy has recent star formation and is on the periphery of the Milky Way: how has it retained its neutral gas reservoir?

The low-mass regime is also where predictions from cosmological simulations and observations of galaxies have the strongest divergence. Much of this can be explained by baryonic processes, but this physics is implemented at the sub-grid level and predictions for low mass galaxies are extremely sensitive to the resolution of the survey, e.g., [3]. Observations of low mass galaxies can be used to address a few key questions to help constrain and interpret results from simulations:

What is the lowest mass galaxy that can form? There is a dearth of observed low-mass galaxies compared to theoretical predictions for the number of low-mass DM halos. In the Local Group, this is the canonical "missing satellites problem". This discrepancy can be explained by baryonic processes resulting in the loss of gas from the lowest mass halos, resulting in DM halos that lack an observable counterpart. An important constraint for the simulations is determining if there an abrupt cutoff in galaxy mass and/or DM halo mass at which there are no longer observable galaxies. As the resolutions of simulations increase, they generally produce lower mass galaxies [3], and at the same time, as observations have increased in sensitivity, new low mass galaxies have been discovered [4]. Observational studies to date have concentrated on satellite galaxies around more massive central galaxies where environmental processes are important. An important step forward is to expand this work to low-mass field galaxies as isolated systems. As these galaxies are typically gas-rich, H I surveys are an excellent means of both identifying and studying these galaxies.

Which DM halos do low mass galaxies live in? The explanation for the lack of low mass galaxies requires that not all DM halos host an observable galaxy. An important test of the simulations that reproduce the required number of galaxies is to observationally confirm that observed dwarf galaxies live in the DM halos that we expect. The naive expectation is that more massive DM halos host galaxies while lower mass ones do not. However, observations appear to show that observed dwarf galaxies live in less massive DM halos than expected (the "too big to fail" problem). This was originally seen in the satellites of the MW [5] but has recently been recognized to occur for field galaxies also; this is important because many of the proposed explanations for this discrepancy rely on interaction with the central galaxy [6]. The kinematics of (marginally) resolved low-mass galaxies can be used to address this observationally, by using rotation curve modelling or simply a last measured velocity and radius to constrain the largest DM halo that could host a galaxy [6]. Identifying low mass galaxies through resolved H I surveys is an efficient observational strategy as the kinematics are provided immediately, and the identified systems are likely field galaxies.

Are low mass galaxies arranged in vast structures around more massive central galaxies? Structures of satellite galaxies have been identified around the Milky Way [7], Andromeda [8] and Cen A [9]. These structures were not predicted from simulations, and it is not clear if they are expected to be common in $\Lambda \mathrm{CDM}$. H I surveys can help address the origin of these structures: are 
they also seen in gas-rich dwarf galaxies? These systems are more isolated and further from central galaxies; this would indicate that these structures may be connected to large scale structure.

\section{Prospectus for current surveys}

Several of the large H I imaging surveys already planned with MeerKAT have the potential to detect and/or resolve galaxies in H I mass ranges of key interest. The three surveys that we consider are MIGHTEE, Fornax and MALS ${ }^{1}$. These three surveys are described elsewhere in this volume; here we briefly summarize a few key parameters for predicting their utility in detecting and resolving low mass galaxies. For comparison, we also consider large H I surveys planned with other SKA pathfinders; these are WALLABY with the Australian SKA Pathfinder (ASKAP), and the Shallow Northern Sky (SNS) and Medium Deep Survey (MDS) with Apertif, a phasedarray feed for the Westerbork Synthesis Radio Telescope. Table 1 summarizes the relevant survey parameters. Figure 1 shows the detection space for the MeerKAT surveys compared with the other surveys. The sensitivity and resolution of MeerKAT allows the planned surveys to detect and resolve low-mass $\mathrm{H}$ I sources to further distances than the surveys planned with other telescopes.

MIGHTEE is a simultaneous continuum and $\mathrm{H}$ I survey of $20 \mathrm{deg}^{2}$. MIGHTEE will have a sensitivity of $\sim 150 \mu \mathrm{Jy}$ beam $^{-1}$ per $26 \mathrm{kHz}$ channel when tapered to a $6.9^{\prime \prime}$ beam. For a linewidth of $20 \mathrm{~km} \mathrm{~s}^{-1}$, this corresponds to a $3-\sigma$ sensitivity of $1.25 \times 10^{20}$ atoms $\mathrm{cm}^{-2}$, or $1 M_{\odot} \mathrm{pc}^{-2}$.

The MeerKAT Fornax Survey is an H I and continuum survey of the Fornax cluster. This survey has a similar sensitivity to MIGHTEE with a footprint of about half the size $\left(12 \mathrm{deg}^{2}\right)$. An important key difference is that this field is specifically chosen because of large scale structure: the Fornax cluster, located at a distance of $\sim 20 \mathrm{Mpc}$.

MALS will be 1000 separate pointings at bright radio sources to search for intervening $\mathrm{H} \mathrm{I}$ and $\mathrm{OH}$ absorption lines. Simultaneously, a shallow, sparsely sampled H I imaging survey will be provided. Since the survey consists of separate pointings, the sensitivity is not uniform. We require a $3-\sigma$ column density limit of $1.25 \times 10^{20}$ atoms $\mathrm{cm}^{-2}$ for a linewidth of $20 \mathrm{~km} \mathrm{~s}^{-1}$. Then, for a $16^{\prime \prime}$ beam, the effective area of MALS is $410 \mathrm{deg}^{2}$. We approximate the point source sensitivity by using the average value between the center of a pointing and the edge set by our column density sensitivity requirement.

WALLABY is a shallow all-sky H I survey with ASKAP. The stated goals of the survey are to survey $31,000 \mathrm{deg}^{2}$ at $30^{\prime \prime}$ resolution with a sensitivity of $1.6 \mathrm{mJy} \mathrm{bm}^{-1}$ in a $4 \mathrm{~km} \mathrm{~s}^{-1}$ channel [11]. For an intrinsic linewidth of $20 \mathrm{~km} \mathrm{~s}^{-1}$, this corresponds to a 3- $\sigma$ column density limit of $5.3 \times 10^{19}$ atoms $\mathrm{cm}^{-2}$. In Table 1 we assume 50\% WALLABY sky coverage; due to a likely decrease in sensitivity the mapping speed of ASKAP will decrease by a factor of $\sim 2$, and this represents that effect. For a full WALLABY survey, the predictions can be multiplied by a factor of two.

The Apertif SNS will cover $\sim 3500 \mathrm{deg}^{2}$ to a similar sensitivity level as WALLABY. The rms noise in a $20 \mathrm{~km} \mathrm{~s}^{-1}$ channel is expected to be $0.65 \mathrm{mJy} \mathrm{bm}^{-1}$ [12]. The Apertif beam depends

\footnotetext{
${ }^{1}$ LADUMA has too small a footprint to probe a local volume where low mass galaxies can be detected/resolved. MHONGOOSE observations do not reach out past the virial radius in most cases, where gas-rich galaxies are expected to be detected [10].
} 


\begin{tabular}{lllll}
\hline \hline Survey & $\begin{array}{l}S_{\text {det }} \\
\begin{array}{l}\Delta \nu=30 \mathrm{~km} \mathrm{~s}^{-1} \\
\mathrm{mJy} \mathrm{km} \mathrm{s}^{-1}\end{array}\end{array}$ & $\theta$ & $\begin{array}{l}3-\sigma N_{H I} \\
\Delta v=20 \mathrm{~km} \mathrm{~s}^{-1}\end{array}$ & FoV \\
& 11 & 6.9 & $1.25 \times 10^{20}$ & 20 \\
aIGHTEE & 11 & 6.9 & $1.25 \times 10^{20}$ & 12 \\
Fornax & 52 & 16 & $1.25 \times 10^{20}$ & 410 \\
MALS & 30 & $5.3 \times 10^{19}$ & $15000^{a}$ \\
WALLABY & 88 & 20 & $1.1 \times 10^{20}$ & 3500 \\
Apertif SNS & 80 & 20 & $4.1 \times 10^{19}$ & 450 \\
Apertif MDS & 31 & 16 & $1.25 \times 10^{20}$ & 300 \\
\hline Centaurus & 36 & & & \\
\hline
\end{tabular}

Table 1: Summary of parameters for currently planned H I surveys.

Columns are: Survey, 5- $\sigma$ detection limit for a $30 \mathrm{~km} \mathrm{~s}^{-1}$ linewidth source (3- $\sigma$ for a $84 \mathrm{~km} \mathrm{~s}^{-1}$ source), beam size in arcseconds, $3-\sigma$ column density limit for a linewidth of $20 \mathrm{~km} \mathrm{~s}^{-1}$, and survey footprint.

${ }^{a}$ This is $50 \%$ of the nominal survey area; see text for a discussion of this choice.

on the declination; for an average $20^{\prime \prime}$ beam this corresponds to a 3- $\sigma$ column density limit of $1.1 \times 10^{20}$ atoms $\mathrm{cm}^{-2}$.

The Apertif MDS is deeper observations of a $\sim 450 \mathrm{deg}^{2}$ region, reaching $0.25 \mathrm{mJy} \mathrm{bm}^{-1}$ for a $20 \mathrm{~km} \mathrm{~s}^{-1}$ channel, or a 3- $\sigma$ column density limit of $4.1 \times 10^{19}$ atoms $\mathrm{cm}^{-2}$. Part of the MDS footprint includes the local overdensity of the Canes Venaciti loose groups, CVn I and CVn II.
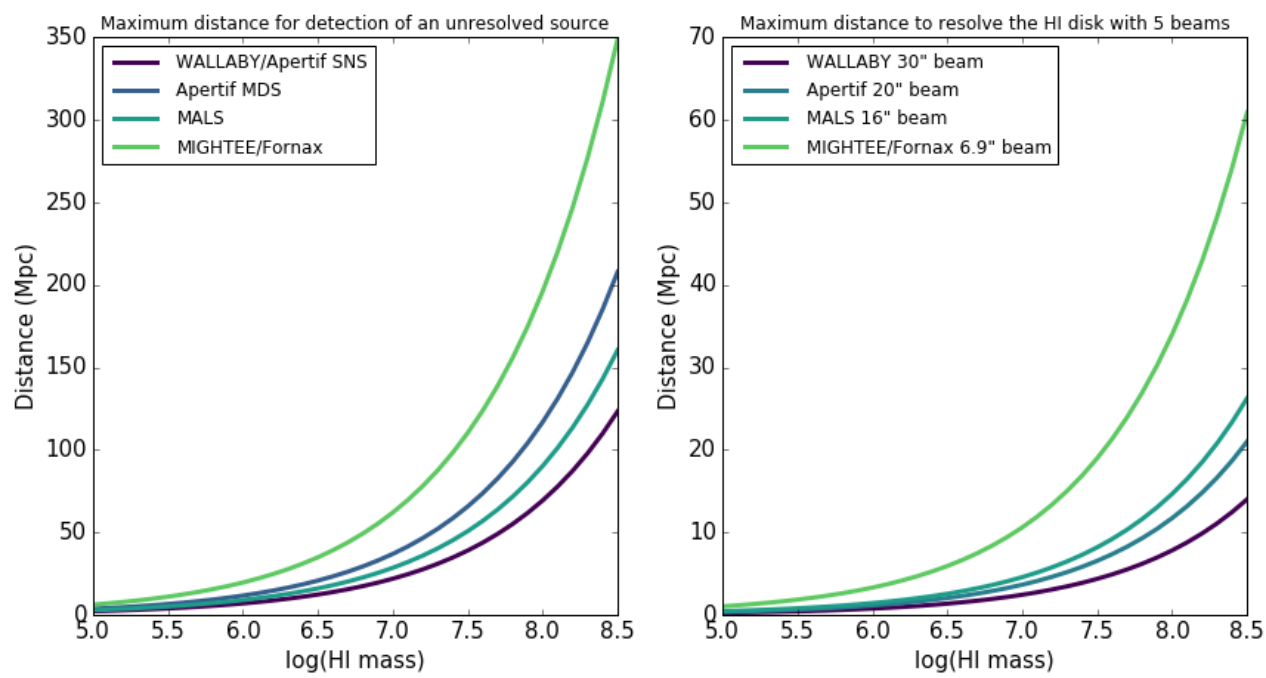

Figure 1: Detection space for MeerKAT surveys and other surveys.

We use the survey parameters to make predictions for the number of sources that we expect the different surveys to both detect and resolve. We focus on two mass regimes of particular interest: (a) $10^{5}<M_{H I}<10^{7} M_{\odot}$ as the lowest mass systems where we wish to determine how many and which galaxies survive and (b) $10^{7}<M_{H I}<10^{8.5} M_{\odot}$ as the mass range where more resolved $\mathrm{H} \mathrm{I}$ kinematics can help address the too big to fail problem [6]. We integrate the H I mass function 
(HIMF), assuming the line flux detection limits in Table 1. There we report the 5- $\sigma$ detection limit for a $30 \mathrm{~km} \mathrm{~s}^{-1}$ linewidth unresolved source; this is the typical width for a galaxy with $M_{\mathrm{HI}} \sim 10^{6}$ $M_{\odot}$. This same limit is the $3-\sigma$ detection limit for a $84 \mathrm{~km} \mathrm{~s}^{-1}$ linewidth source, a velocity width more typical for galaxies of $\sim 10^{8} M_{\odot}$. In general we use the HIMF from the ALFALFA H I survey as a representative field HIMF [13]. For the Apertif MDS we also use the CVn HIMF [14] for the relevant part of the volume to properly account for the known overdensity. The CVn HIMF has a higher normalization than the ALFALFA HIMF as it represents an overdense region of space. At the same time, the slope of the low mass end is flatter, likely due to processing by the environment and loss of $\mathrm{H}$ I from some of the galaxies. Fornax includes an overdensity, but, as a cluster, is an even more extreme environment where fragile low mass $\mathrm{H}$ I objects are likely to be destroyed. Determining the HIMF of Fornax is a science goal of that survey. We use the ALFALFA field HIMF as an approximation. Because it does not account for the known overdensity, more low mass $\mathrm{H}$ I sources are likely be detected than we predict.

In order to determine how many resolved objects are expected to be detected, we again integrate the appropriate HIMF but set distance limits for each mass bin based on how far out an object can be resolved, rather than to where it can be detected. In order to set these distance limits, we use the H I mass-diameter relation [15], a tight correlation between the H I mass of a galaxy and its diameter at the $1 M_{\odot} \mathrm{pc}^{-2}\left(1.25 \times 10^{20}\right.$ atoms $\left.\mathrm{cm}^{-2}\right)$ level. For the roughest constraint of the resolved kinematics of galaxies, we ask for 3 beams across a galaxy. This is not enough for detailed kinematic modelling but may be enough to constrain a maximum velocity and the extent at which occurs, allowing a constraint on the largest DM halo that may host a galaxy following the methodology of [6]. We also predict how many systems will be resolved by five or more beams; these can be more fully modelled.

Table 2 reports our predictions. We note that these numbers should serve as a rough guide only. We do not account for any scatter in the H I mass-diameter relation or for cosmic variance (except to discard systems with $D<3 \mathrm{Mpc}$; objects at this distance or closer may be at low velocities that are confused with foreground Milky Way H I emission). This latter point point is especially important. MIGHTEE and Fornax have small footprints and so the structure along the line of sight will heavily influence the final number of detections. The other surveys are relatively shallow and so can only detect and resolve low mass galaxies at small distances, and structure in the local Universe will strongly change their predictions. Figure 2 depicts the comparison in the number of expected detected and resolved ( $>5$ beams) sources for the various surveys. While the MeerKAT surveys are more sensitive and have better resolution, the shallow surveys planned with other telescopes are expected to detect (and resolve) more objects due to their large footprint. The MeerKAT surveys may detect as many $\sim 60$ sources with $\mathrm{H}$ I masses below $10^{7} M_{\odot}$, approximately double the number of sources in this mass range in the literature. Given the rough value of these estimates, the MeerKAT surveys should at least provide a sample of the lowest mass H I-selected dwarfs comparable to samples currently available. The surveys will also well resolve $\sim 15$ objects in the mass range $10^{7}-10^{8.5} M_{\odot}$, an important parameter space for determining which DM halos host observable galaxies. A further $\sim 60$ objects will be marginally resolved, which may provide enough information to constrain their hosting DM halo. 


\begin{tabular}{llllllll}
\hline \hline & & \multicolumn{3}{c}{$10^{5}<M_{H I}<10^{7} M_{\odot}$} & \multicolumn{3}{c}{$10^{7}<M_{H I}<10^{8.5} M_{\odot}$} \\
Survey & HIMF & $N_{\text {det }}$ & $N_{3 \text { beams }}$ & $N_{5 \text { beams }}$ & $N_{\text {det }}$ & $N_{3 \text { beams }}$ & $N_{\text {5beams }}$ \\
\hline MIGHTEE & ALFALFA & 19 & 0.4 & 0.1 & 1000 & 25 & 5 \\
Fornax & ALFALFA $^{a}$ & 11 & 0.3 & 0.1 & 620 & 15 & 3 \\
MALS & ALFALFA & 37 & 0.5 & 0 & 2100 & 41 & 9 \\
WALLABY & ALFALFA & 610 & 0.7 & 0 & 34000 & 220 & 46 \\
SNS & ALFALFA & 156 & 2 & 0.1 & 9300 & 180 & 38 \\
MDS & ALFALFA + CVn & 180 & 1.4 & 0.3 & 5000 & 70 & 27 \\
\hline Centaurus & CVn + ALFALFA & $63(18)$ & $3(3)$ & $0.5(0.5)$ & $2600(9)$ & $38(9)$ & $13(8)$ \\
\hline
\end{tabular}

Table 2: Expectations for detections of low-mass galaxies for currently planned H I surveys. For the proposed Centaurus survey, numbers in parentheses indicate the number of detections in only the Centaurus region, not including background sources.

${ }^{a}$ Fornax is an overdense environment and the use of the ALFALFA HIMF is a conservative choice; see text for a further discussion.
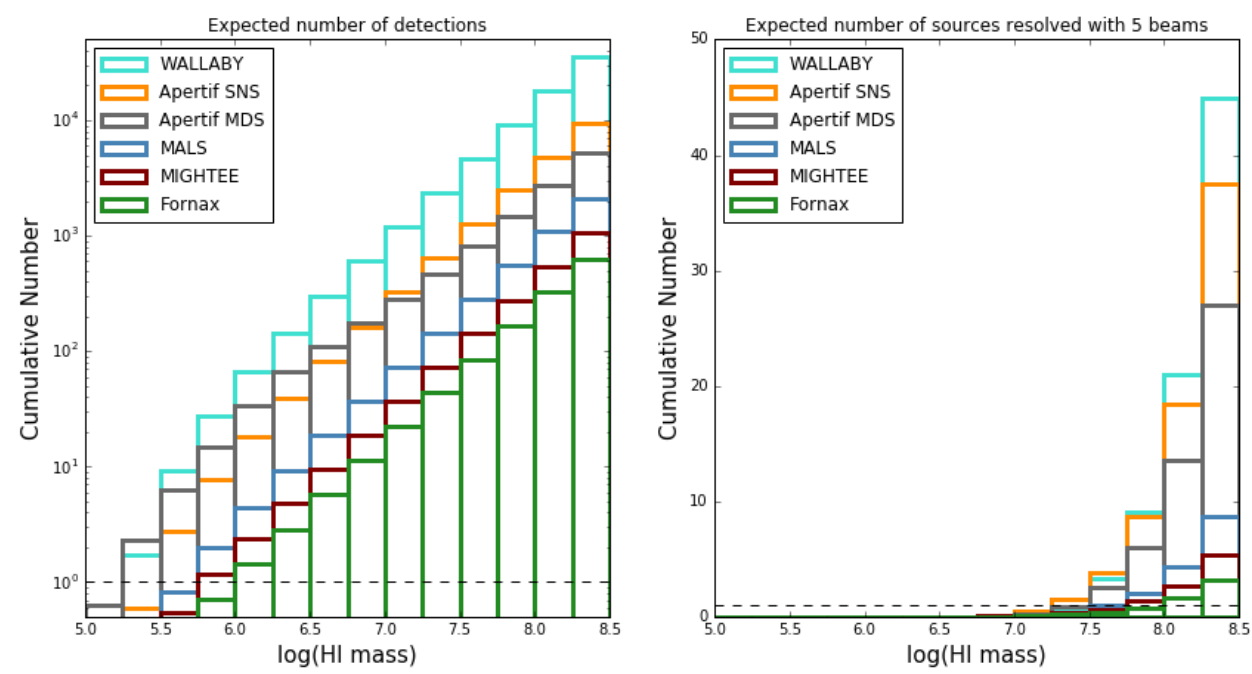

Figure 2: Comparison of expected number of detections and resolved sources ( $>5$ beams) at $D>3 \mathrm{Mpc}$ for the various H I surveys.

\section{A dedicated MeerKAT project for the smallest galaxies}

MeerKAT has the sensitivity and resolution to greatly advance studies of the lowest mass gas-rich galaxies. The planned large surveys will produce a sample of the lowest-mass H I-rich dwarf galaxies and a sample of resolved dwarf galaxy kinematics comparable to currently existing samples. However, this is not the prime focus of any of these surveys. Here we propose a 1000 hour survey ( 850 hours of on-source time) that would have a lasting legacy.

\subsection{Proposed survey}

We propose a survey of the Centaurus region, encompassing the M 83 and Cen A galaxy 
groups. These are among the closest galaxy groups to us; the Cen A group is at a distance of 3.76 $\mathrm{Mpc}$, and the M83 group is at a distance of $4.79 \mathrm{Mpc}$ [16]. The left panel of Figure 3 shows the distribution of distances for galaxies in this structure. Importantly for $\mathrm{H} \mathrm{I}$ studies, these groups have mean recessional velocities that are clearly distinct from foreground Milky Way H I emission; the distribution of velocities of galaxies in these groups are shown in the right panel of Figure 3. This both allows the detection of $\mathrm{H}$ I sources without confusion with Galactic $\mathrm{H}$ I and the assignment of a group distance without any optical measurements.
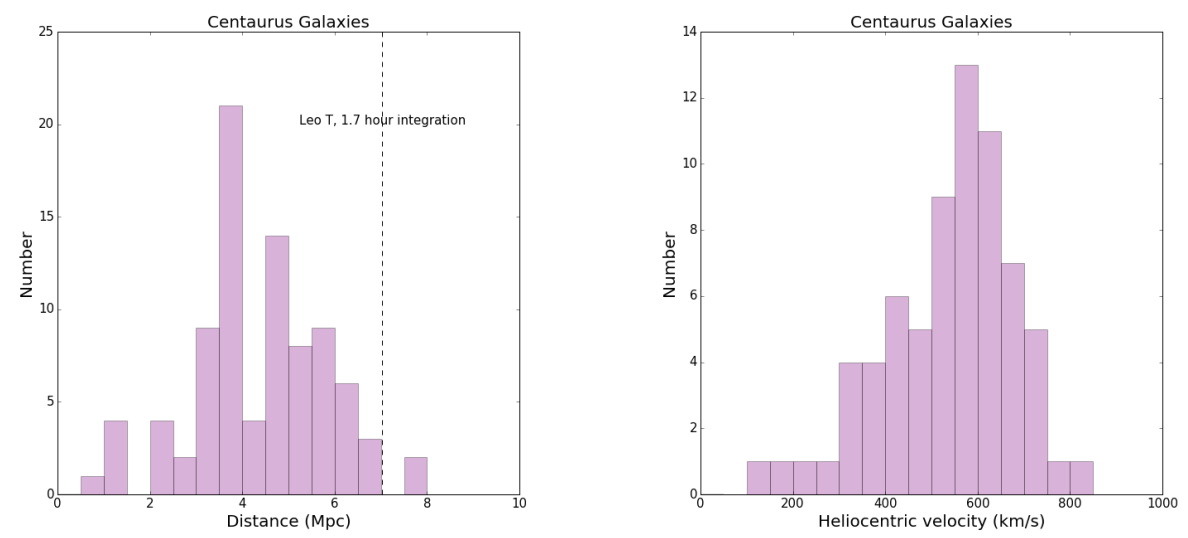

Figure 3: Left: Histogram of distances of galaxies in Centaurus from [16]. Right: Histogram of velocities of galaxies in Centaurus from [16]. The systems are well separated from Galactic emission and systems with velocities of $\sim 600 \mathrm{~km} \mathrm{~s}^{-1}$ can be directly associated with Centaurus without a distance measure.

A core requirement for this survey is the ability to detect an object similar to Leo T over the volume of the group. The H I mass of Leo T is $4.2 \times 10^{5} M_{\odot}$ [1], and the group edge is at $7 \mathrm{Mpc}$. This corresponds to an $\mathrm{H} \mathrm{I}$ flux of $36.3 \mathrm{mJy} \mathrm{km} \mathrm{s}^{-1}$. To calculate the time required to detect such a source, we take $30 \mathrm{~km} \mathrm{~s}^{-1}$ as a maximum linewidth for such a low-mass object (the linewidth of Leo $\mathrm{T}$ is $\sim 20 \mathrm{~km} \mathrm{~s}^{-1}$ ). Using the updated specifications for MeerKAT (SEFD=424 Jy), and assuming a 1.5 loss of sensitivity for a robust weighting scheme, we find that we require 1.7 hours of on-source integration time for a 5- $\sigma$ detection.

An important constraint is that we wish to be sensitive to the main $\mathrm{H} \mathrm{I} \mathrm{disk} \mathrm{of} \mathrm{resolved} \mathrm{systems.}$ We require that we be able to detect the column density level of $1.25 \times 10^{20}$ atoms $\mathrm{cm}^{-2}\left(1 \mathrm{M}_{\odot}\right.$ $\mathrm{pc}^{-2}$ ) at the $3-\sigma$ level for an intrinsic linewidth of $20 \mathrm{~km} \mathrm{~s}^{-1}$. For an on-source integration time of 1.7 hours, this implies a $16^{\prime \prime}$ beam or larger be used for imaging.

We consider a survey footprint of $300 \mathrm{deg}^{2}$. This is a large enough survey footprint to extend well beyond the virial radii of M 83 and Cen A, where we expect to detect few gas-rich galaxies [10], and to map the region between the two subgroups. This footprint is shown in Figure 4. As discussed in the previous section, a large survey footprint is critical for increasing the number of low-mass sources detected. In order to calculate the total time for the survey, we assume an effective field of view of $0.6 \mathrm{deg}^{2}$ for each pointing. Then, it takes 850 hours of on-source integration time (or $\sim 1000$ hours total including overhead) to map the Centaurus region to the required sensitivity. The parameters of this proposed survey are included in Table 1. 


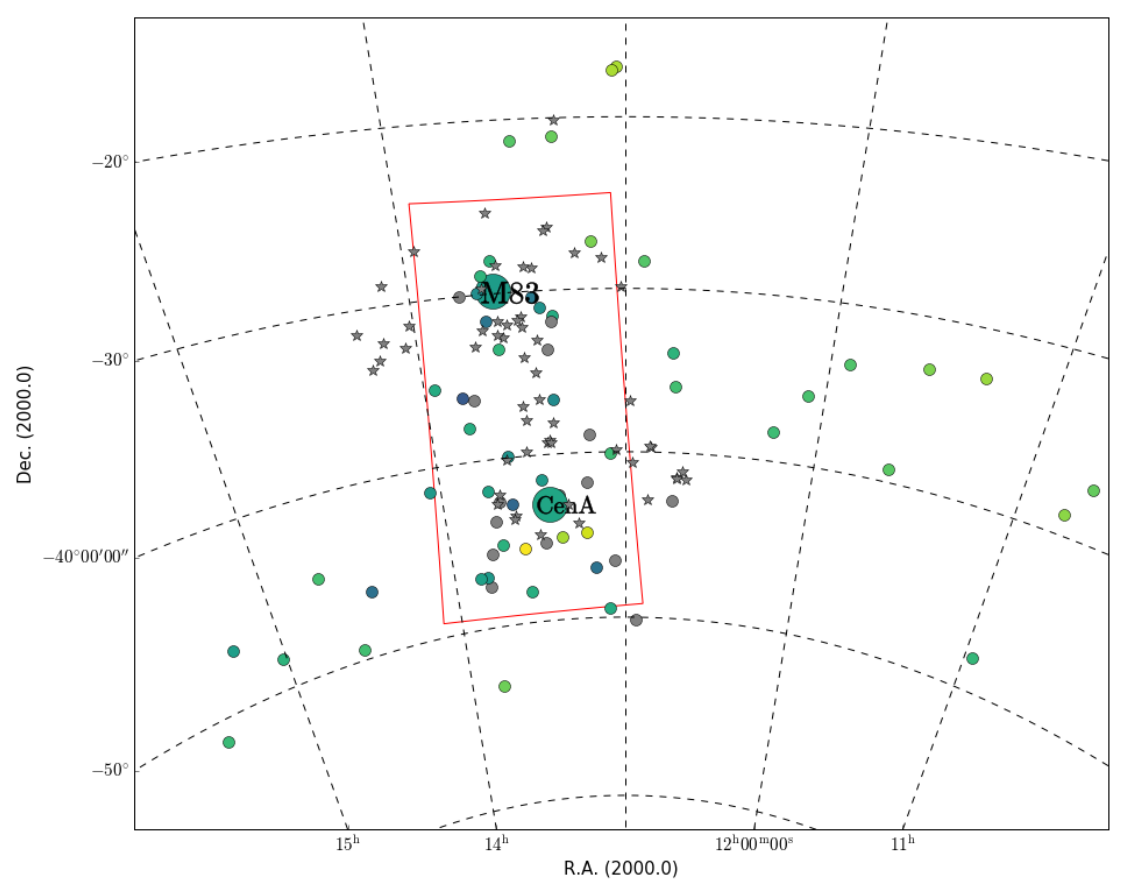

Figure 4: Galaxies in the Centaurus group from [16] (circles) plus potential dwarf galaxy members (gray stars) from $[17,18]$. The proposed $300 \mathrm{deg}^{2}$ footprint is shown by the red box.

\subsection{Expectations for the survey}

Given our sensitivity limit, beam size, and footprint area, we can predict how many sources we expect to detect and resolve. We use the same mechanisms as in the previous section where we integrate the HIMF using our survey parameters. As this survey specifically targets an overdense region, we use the CVn HIMF for the distance range of the Centaurus group, 2-7 Mpc. We also make predictions for the detection of background sources at distances greater than $7 \mathrm{Mpc}$ using the ALFALFA HIMF. Figure 5 shows our predictions for the proposed survey. In the left panel we show the predictions for the Centuaurs region only. In the right panel we compare the complete Centaurus survey (including background sources) to other planned surveys. We also report the number of sources we expect to detect in Table 2.

\subsection{Key science of the survey}

This proposed survey of the Centaurus region would detect and resolve a similar number of galaxies as large-field surveys with other telescopes. However, one key difference is that any detected H I sources at velocities below $800 \mathrm{~km} \mathrm{~s}^{-1}$ can be associated with Centaurus and thus have a group distance without optical follow-up. Good distances are key for studies of low mass galaxies [19]. Key science questions that will be addressed by this survey are:

How many galaxies like Leo T exist in a typical galaxy group? By design, this survey will be able to detect an object with the $\mathrm{H}$ I mass of Leo $\mathrm{T}$ throughout the Centaurus region. Leo $\mathrm{T}$ is on edge of detectability for SDSS, and its H I content is almost confused in velocity-space with the Galactic foreground. Since the discovery of Leo T, further optical and H I searches have only 
uncovered one similar system, Leo $\mathrm{P}$, which has an H I mass of $8.1 \times 10^{5} M_{\odot}$, a stellar mass of $5.6 \times 10^{5} M_{\odot}$, and sits just outside the Local Group at a distance of $1.62 \mathrm{Mpc}$ [20]. Given the observational difficulties in detecting these systems in our own galaxy group and the fact that Leo $\mathrm{P}$ was discovered as an $\mathrm{H}$ I source [21], the best way to determine how common marginal systems like Leo $\mathrm{T}$ are may be by surveying an external galaxy group in $\mathrm{H} \mathrm{I}$.

How is the gas distributed in these systems and what is the potential for star formation? At a median distance of $4 \mathrm{Mpc}$, a $16^{\prime \prime}$ beam corresponds to a linear resolution of $300 \mathrm{pc}$. This is comparable to the physical size of Leo $\mathrm{T}$ but would allow more massive systems to be (slightly) resolved. MeerKAT data has the large advantage that it can be imaged at multiple spatial scales. Higher resolution maps can be produced, at the expense of column density sensitivity, in order to localize high column density emission. In addition, deeper follow-up observations with MeerKAT of the most intriguing low-mass systems can be undertaken in order to image the $\mathrm{HI}$ at higher angular resolution. Both Leo $\mathrm{T}$ and Leo $\mathrm{P}$ have a cool neutral medium component, evidence for cooler dense gas and the potential for star formation [22,23]. It this common at this mass scale?

Do the satellite structures extend to the field? Recent observations show that the satellite galaxies around Cen A are arranged in a plane [24]. With the addition of $\mathrm{H}$ I observations, we can see if this structure extends to gas-rich galaxies that are more removed from Cen A. If this structure extends further, that is a clue that it may be related to large scale structure.

What halos do low mass galaxies live in? We expect to well resolve $\sim 10$ sources in the Centaurus region below $10^{8.5} M_{\odot}$. These galaxies can serve as an important comparison sample for other H I field surveys as they live in a denser environment. Does environment affect our predictions for the halos that host galaxies of a given mass even when looking at an $\mathrm{H}$ I rich population?
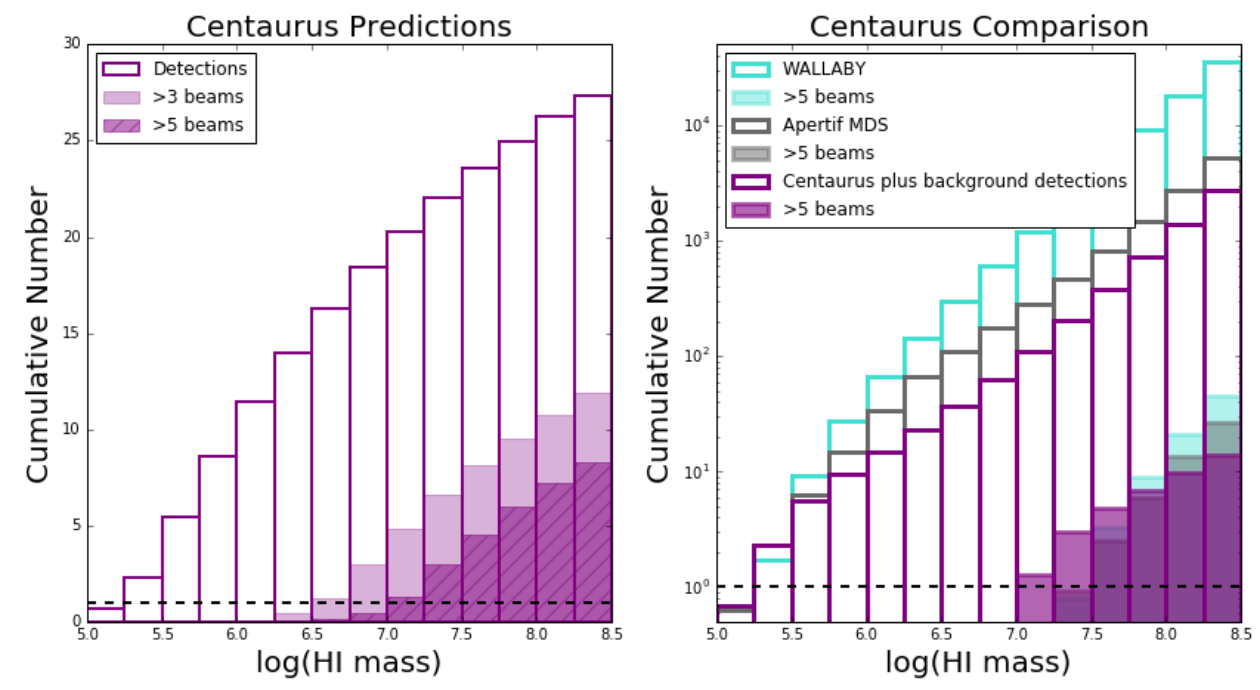

Figure 5: Predictions for number of detections in the Centaurus tregion.

\section{Conclusions}

Studies of the smallest galaxies offer critical tests for cosmology and galaxy formation. Cur- 
rently planned MeerKAT surveys have the potential to double the number of low-mass dwarf galaxies with $M_{\mathrm{HI}}<10^{7} M_{\odot}$. They will also provide a number of (marginally) resolved systems below $10^{8.5} M_{\odot}$ comparable to current samples in the literature. The resolved $\mathrm{H}$ I data allow a constraint on halos that galaxies live in which can be compared to results from abundance matching. This is a significant increase in the number of known and resolved systems in these mass ranges, but not competitive with H I surveys planned for other SKA pathfinders. We propose a survey of the Centaurus region, including the M 83 and Cen A galaxy groups, which would robustly address questions on low-mass galaxies, notably: "How common are systems like Leo T?". This survey would also play an important role in the recent, open question of satellite galaxies forming vast structures; the H I observations would reveal if dwarf galaxies continue to from this structure further from the central galaxy, implying that it may be connected to large scale structure.

\section{Acknowledgments}

We thank Kristine Spekkens for helpful discussion on the sensitivity limits of the various H I survey and Paolo Serra for plots showing the effect of various weighting and tapering schemes on the sensitivity and restoring beam shape of MeerKAT.

\section{References}

[1] E. A. K. Adams and T. A. Oosterloo, Deep WSRT HI observations of Leo T, in preparation (2016) .

[2] D. R. Weisz, D. B. Zucker, A. E. Dolphin, N. F. Martin, J. T. A. de Jong, J. A. Holtzman et al., The Star Formation History of Leo T from Hubble Space Telescope Imaging, ApJ 748 (Apr., 2012) 88, [1201.4859].

[3] C. Wheeler, J. Oñorbe, J. S. Bullock, M. Boylan-Kolchin, O. D. Elbert, S. Garrison-Kimmel et al., Sweating the small stuff: simulating dwarf galaxies, ultra-faint dwarf galaxies, and their own tiny satellites, MNRAS 453 (Oct., 2015) 1305-1316, [1504.02466].

[4] A. Drlica-Wagner, K. Bechtol, E. S. Rykoff, E. Luque, A. Queiroz, Y.-Y. Mao et al., Eight Ultra-faint Galaxy Candidates Discovered in Year Two of the Dark Energy Survey, ApJ 813 (Nov., 2015) 109, [1508.03622].

[5] M. Boylan-Kolchin, J. S. Bullock and M. Kaplinghat, The Milky Way's bright satellites as an apparent failure of $\Lambda C D M$, MNRAS 422 (May, 2012) 1203-1218, [1111.2048].

[6] E. Papastergis, R. Giovanelli, M. P. Haynes and F. Shankar, Is there a "too big to fail" problem in the field?, A\&A 574 (Feb., 2015) A113, [1407.4665].

[7] M. S. Pawlowski and P. Kroupa, The Vast Polar Structure of the Milky Way Attains New Members, ApJ 790 (July, 2014) 74, [1407.2612].

[8] R. A. Ibata, G. F. Lewis, A. R. Conn, M. J. Irwin, A. W. McConnachie, S. C. Chapman et al., A vast, thin plane of corotating dwarf galaxies orbiting the Andromeda galaxy, Nature 493 (Jan., 2013) 62-65, [1301.0446].

[9] R. B. Tully, N. I. Libeskind, I. D. Karachentsev, V. E. Karachentseva, L. Rizzi and E. J. Shaya, Two Planes of Satellites in the Centaurus A Group, ApJ 802 (Apr., 2015) L25, [1503. 05599].

[10] K. Spekkens, N. Urbancic, B. S. Mason, B. Willman and J. E. Aguirre, The Dearth of Neutral Hydrogen in Galactic Dwarf Spheroidal Galaxies, ApJ 795 (Nov., 2014) L5, [1410 . 0028]. 
[11] B. S. Koribalski, Overview on Spectral Line Source Finding and Visualisation, PASA 29 (Aug., 2012) 359-370, [1206.6916].

[12] Apertif Science Team, Apertif Survey Plan, http://www.astron.nl/sites/astron.nl/files/cms/OTHER/ApertifSurveyPlanII.v2.2.pdf (Mar., 2016) .

[13] A. M. Martin, E. Papastergis, R. Giovanelli, M. P. Haynes, C. M. Springob and S. Stierwalt, The Arecibo Legacy Fast ALFA Survey. X. The H I Mass Function and $\Omega \_H$ I from the 40\% ALFALFA Survey, ApJ 723 (Nov., 2010) 1359-1374, [1008.5107].

[14] K. Kovač, T. A. Oosterloo and J. M. van der Hulst, A blind HI survey in the Canes Venatici region, MNRAS 400 (Dec., 2009) 743-765, [0 904.2775$].$

[15] J. Wang, B. S. Koribalski, P. Serra, T. van der Hulst, S. Roychowdhury, P. Kamphuis et al., New lessons from the H I size-mass relation of galaxies, MNRAS 460 (Aug., 2016) 2143-2151, [1605.01489].

[16] I. D. Karachentsev, R. B. Tully, A. Dolphin, M. Sharina, L. Makarova, D. Makarov et al., The Hubble Flow around the Centaurus A/M83 Galaxy Complex, lAJ 133 (Feb., 2007) 504-517, [astro-ph/0603091].

[17] O. Müller, H. Jerjen and B. Binggeli, New dwarf galaxy candidates in the Centaurus group, A\&A 583 (Nov., 2015) A79, [1509.04931].

[18] O. Müller, H. Jerjen and B. Binggeli, New low surface brightness dwarf galaxies in the Centaurus group, ArXiv e-prints (May, 2016) , [1605.04130].

[19] K. B. W. McQuinn, J. M. Cannon, A. E. Dolphin, E. D. Skillman, J. J. Salzer, M. P. Haynes et al., Distance Determinations to SHIELD Galaxies from Hubble Space Telescope Imaging, ApJ 785 (Apr., 2014) 3, [1402.3723].

[20] K. B. W. McQuinn, E. D. Skillman, A. Dolphin, J. M. Cannon, J. J. Salzer, K. L. Rhode et al., Leo P: An Unquenched Very Low-mass Galaxy, ApJ 812 (Oct., 2015) 158, [1506. 05495 ].

[21] R. Giovanelli, M. P. Haynes, E. A. K. Adams, J. M. Cannon, K. L. Rhode, J. J. Salzer et al., ALFALFA Discovery of the Nearby Gas-rich Dwarf Galaxy Leo P. I. H I Observations, lAJ 146 (July, 2013) 15, [1305.0272].

[22] E. V. Ryan-Weber, A. Begum, T. Oosterloo, S. Pal, M. J. Irwin, V. Belokurov et al., The Local Group dwarf Leo T: HI on the brink of star formation, MNRAS 384 (Feb., 2008) 535-540, [0711. 2979].

[23] E. Z. Bernstein-Cooper, J. M. Cannon, E. C. Elson, S. R. Warren, J. Chengular, E. D. Skillman et al., ALFALFA Discovery of the Nearby Gas-rich Dwarf Galaxy Leo P. V. Neutral Gas Dynamics and Kinematics, lAJ 148 (Aug., 2014) 35, [1404.5298].

[24] O. Müller, H. Jerjen, M. S. Pawlowski and B. Binggeli, Testing the two planes of satellites in the Centaurus Group, ArXiv e-prints (July, 2016), [1607.04024]. 